\title{
Long non-coding RNA RP11-465L10.10 promotes vascular smooth muscle cells phenotype switching and MMP9 expression via the NF-кB pathway
}

\author{
Yang Lin ${ }^{1 \#}$, Haoyue Huang ${ }^{1,2 \#}$, You Yu ${ }^{1,2 \#}$, Feng Zhu ${ }^{1}$, Weizhang Xiao ${ }^{1}$, Ziying Yang ${ }^{1,2}$, Lianbo Shao ${ }^{1,2 *}$, \\ Zhenya Shen ${ }^{1,2 *}$
}

${ }^{1}$ Department of Cardiovascular Surgery, the First Affiliated Hospital of Soochow University, Suzhou, China; ${ }^{2}$ Institute for Cardiovascular Science, Soochow University, Suzhou, China

Contributions: (I) Conception and design: L Shao, Z Shen, H Huang; (II) Administrative support: Z Shen; (III) Provision of study materials or patients: Z Shen, H Huang; (IV) Collection the tissues from patients: F Zhu, W Xiao; (V) Data analysis and interpretation: Y Lin, L Shao; (VI) Manuscript writing: All authors; (VII) Final approval of manuscript: All authors.

"These authors contributed equally to this work.

*These authors contributed equally to this work.

Correspondence to: Zhenya Shen; Lianbo Shao. Department of Cardiovascular Surgery, the First Affiliated Hospital of Soochow University, No. 899 Pinghai Road, Suzhou 215006, China. Email: uuzyshen@aliyun.com; shaolianbo1987@126.com.

Background: Thoracic aortic aneurysm/dissection (TAA/D) are complicated vascular disorders with rapid development and high mortality. Vascular smooth muscle cells (VSMCs) phenotype switching plays an important role in the pathological process of TAA/D. Previous studies have indicated a potential correlation between long non-coding RNA (lncRNA) RP11-465L10.10 and matrix metallopeptidase 9 (MMP9) involved in the development of TAA/D. This study aims to investigate the role of lncRNA RP11-465L10.10 in VSMCs phenotype switching and the molecular mechanism in regulating MMP9 expression.

Methods: The expression of RP11-465L10.10 in vascular tissues and in VMSCs was detected by RTqPCR. To investigate the role of RP11-465L10.10 on VSMCs phenotype switching, an RP11-465L10.10overexpressed lentiviral vector was constructed and transfected into VSMCs. Through EdU staining, migration assay, flow cytometry analysis, the roles of RP11-465L10.10 were estimated. Bioinformatics indicated that RP11-465L10.10 upregulating MMP9 expression via NF- $\mathrm{BB}$ signaling, and SN50 (a specific inhibitor of $\mathrm{NF}-\kappa \mathrm{B}$ pathway) was used to inhibit the $\mathrm{NF}-\kappa \mathrm{B}$ pathway activation, then the expression of MMP9 was detected in RP11-465L10.10 overexpressed VMSCs.

Results: In this study, we found RP11-465L10.10 and MMP9 were highly increased in TAD patient tissues, which was consistent in angiotensin II-induced VSMCs phenotype switching. RP11-465L10.10 overexpression facilitated VSMCs phenotype switching and MMP9 expression. Mechanismly, NF- $\kappa \mathrm{B}$ signal pathway was involved in RP11-465L10.10 induced VSMCs phenotype switching and MMP9 expression by transcriptome data analysis and experimental confirm.

Conclusion: This study demonstrated that RP11-465L10.10 induces VSMCs phenotype switching and MMP9 expression via the NF- $\mathrm{B}$ signal pathway, suggesting that RP11-465L10.10 might be a potential therapeutic target for TAA/D treatment.

Keywords: RP11-465L10.10; vascular smooth muscle cells (VSMCs); phenotype switching; NF-кB pathway; matrix metallopeptidase 9 (MMP9)

Submitted Oct 22, 2021. Accepted for publication Dec 17, 2021.

doi: 10.21037/atm-21-6402

View this article at: https://dx.doi.org/10.21037/atm-21-6402 


\section{Introduction}

Thoracic aortic aneurysm and dissection (TAA/D), caused by aortic vascular smooth muscle cells (VSMCs) loss and extracellular matrix (ECM) degradation, are complex vascular disorders with rapid development and high mortality $(1,2)$. Increasing evidence has demonstrated that VSMCs phenotype switching plays a central role in the pathophysiologic development and progression of TAA/ D (3-5). However, no effective medications have been shown to clinically prevent aortic degeneration and disease progression, due to the lack of understanding about the signaling mechanisms involved in VSMCs phenotypic switch.

Long noncoding RNAs (lncRNA) are a new class of ncRNAs, which function as powerful mediators in multiple aspects of molecular regulation under physiological and pathological conditions (6-8). They play a critical role in regulating tissue inflammation and destruction in aortic vascular disorder. Sun et al. reported that lncRNA H19 promotes vascular inflammation and abdominal aortic aneurysm formation, acting as a competing endogenous RNA (9). Recent research has shown that lncRNA XR007793 regulates cell proliferation and migration via the miR-23b/PPAR $\alpha$ axis in Sprague Dawley (SD) rats aortic VSMCs induced with platelet-derived growth factor (PDGF)-BB (10). The lncRNA MEG8 was first identified as a novel post-transcriptional regulator of VSMCs proliferation and migration by sponging the miR-181a/ PPAR $\alpha$ axis in atherosclerosis (11).

The lncRNA RP11-465L10.10, mapped to human chromosome 20 , is a natural antisense lncRNA transcribed from the downstream of exon 9 of the matrix metallopeptidase 9 (MMP9) gene. Long et al. reported that RP11-465L10.10 presented consistent expression trend with $M M P 9$ and participated in the pathogenesis of early onset pre-eclampsia (EOPE), but the mechanism was not explained (12). Li et al. reported that RP11-465L10.10 is upregulated in TAA by lncRNA expression profile microarray analysis (13). However, whether lncRNAs regulate the activation in VSMCs phenotype switching remains unknown. Recently, it was revealed that the expression of MMP9 is increased in secretory VSMCs (14). Matrix metalloproteinases (MMPs) are important extracellular matrix components and regulate the structure and function of the extracellular matrix, which are initially synthesized in cells and secreted as precursor proteins. MMP9 is one of the matrix metalloproteinases closely related to cardiovascular diseases in the ECM (15). Studies have shown that MMP9 up-regulated in thoracic aortic dissection, promotes the degradation of ECM, destroys the dynamic balance of VSMCs secreting ECM, and participates in the occurrence of TAA/D (16). Based on these correlated and important findings, the significant role of RP11-465L10.10 in regulating VSMCs phenotype switching and $M M P 9$ expression was evaluated in this study. By analyzing the transcriptome data, the underlying mechanism was also explored.

We present the following article in accordance with the MDAR reporting checklist (available at https://dx.doi. org/10.21037/atm-21-6402).

\section{Methods}

\section{Tissue collection}

The study was conducted in accordance with the Declaration of Helsinki (as revised in 2013). This study was approved by the Institutional Review Board of the First Affiliated Hospital of Soochow University (No. 2021-305). Written informed consents were provided by individual participants or their guardians. Tissues analyzed in this study were collected from the Department of Cardiovascular Surgery, the First Affiliated Hospital of Soochow University. Aortic dissection specimens were obtained during the Bentall procedures or other large vessel replacements of TAD patients. Patients with traumatic aortic injury, inflammatory aortic disease, Ehlers-Danlos syndrome, Marfan syndrome, and other connective tissue disorders were excluded (17). Control aortic specimens were obtained from patients who underwent aortic valve replacement without vascular wall lesion. Specimens were divided into pieces and preserved under different conditions for histological and biochemical analysis.

\section{Immunohistochemical staining}

Tissues for histologic analysis were embedded in paraffin and sectioned at $5 \mu \mathrm{m}$ thickness. After dewaxing, rehydration, and antigen retrieval, the sections were incubated with anti-MMP9 antibody (1:500) overnight at $4{ }^{\circ} \mathrm{C}$, and then incubated with horseradish peroxidase (HRP)-conjugated secondary antibody for $1 \mathrm{~h}$ at room temperature. Finally, a 3,3-diaminobenzidine (DAB) horseradish peroxidase color development kit (Servicebio, Wuhan, China) was used for color development. 


\section{Cell culture}

Human primary aortic VSMCs (BNCC 338291) were obtained from the Bena Culture Collection and cultured in Dulbecco's modified Eagle medium (DMEM)/high glucose medium containing $10 \%$ fetal bovine serum (FBS) and $1 \%$ penicillin/streptomycin. Only 4-8 passages of VSMCs were used in the experiments.

\section{Overexpression RP11-465L10.10 in VSMCs}

The DNA sequence fragment of RP11-465L10.10 was synthesized by GENEWIZ Company (Suzhou, China), and cloned into a $\mathrm{pCDH}$ lentivirus vector, which contained a green fluorescence protein (GFP) tag. Then, the lentivirus particles were produced in 293 T cells and enriched with PEG8000. The VSMCs were infected with the lentivirus particles. After 3 days, the GFP positive cells were sorted with a flow sorting system (Becton, Dickinson, and Co., Franklin Lakes, NJ, USA).

\section{Total RNA extraction and reverse transcription polymerase chain reaction}

Total RNA was extracted from VSMCs with RNAiso Plus reagent (Takara, Dalian, China) according to the manufacturer's instruction. Prime script RT Reagent (Takara, Dalian, China) was used to reverse-transcribe $1,000 \mathrm{ng}$ of total RNA into complementary DNA (cDNA). Gene expression was detected by FastStart Universal SYBR-Green Master (Takara, Dalian, China). Primers used in this study were designed by primer-BLAST of National Center for Biotechnology Information (NCBI) and are shown in Table S1. Relative fold changes of genes expression were normalized to $18 \mathrm{~s}$ RNA and calculated by the $2^{-\Delta \Delta \mathrm{Ct}}$ method.

\section{Cell migration assay}

Migration assay was performed with VSMCs following published methods (18). Briefly, RP11-465L10.10 differently expressed VSMCs and negative control group were seeded in a 6 -well plate $\left(6 \times 10^{5}\right.$ cells/well $)$ and grown to $80 \%$ confluence, a linear scratch was made with a $200-\mu \mathrm{L}$ pipette tip. Then, the cells were incubated with DMEM/ high glucose basic medium for specific times. Images were taken at $24 \mathrm{~h}$ to measure migration ratios.

\section{Cell proliferation assay}

The capability of cell proliferation was detected by 5-ethynyl-2'-deoxyuridine (EdU) incorporation assay. The RP11-465L10.10 differently expressed VSMCs were seeded in 24-well plates with $1.5 \times 10^{5}$ cells per well and cultured for $24 \mathrm{~h}$, then cocultured with EdU for $2 \mathrm{~h}$ at $37^{\circ} \mathrm{C}$. Cells were fixed and permeabilized, followed by staining with active click buffer for $30 \mathrm{~min}$ protected from light. Subsequently, nuclei were stained with Hoechst 33342 (Beyotime, Shanghai, China) for $30 \mathrm{~min}$ protected from light. Finally, the images were obtained with a fluorescent microscope and quantitatively analyzed.

\section{Cell apoptosis assay}

Apoptosis was analyzed by flow cytometry. After being induced with $500 \mu \mathrm{M} \mathrm{H}_{2} \mathrm{O}_{2}$ for $12 \mathrm{~h}$, VSMCs were stained with the Annexin V-PE/7-AAD apoptosis flow cytometry detection kit (Becton, Dickinson, and Co., Franklin Lakes, NJ, USA) following the manufacture's protocol. Meanwhile, nuclear morphology was stained with 4',6-diamino-2phenylindole (DAPI).

\section{RNA-Seq analysis in RP11-465L10.10(+) group}

The RNA expression profiles analysis of the RP11465 L10.10 overexpression group and the control group were performed by Novogene Company (Beijing, China). The total RNA of VSMCs was extracted with RNAiso Plus reagent (Takara, Dalian, China), RNA quantification and qualification RNA integrity were assessed using the RNA Nano 6000 Assay Kit of the Bioanalyzer 2100 system (Agilent Technologies, Santa Clara, CA, USA). The transcriptome library preparation and sequencing were performed according to library preparation for transcriptome sequencing. Triplicates of each group were analyzed. Differentially detected signals were identified through fold change filtering [fold change (FC) $\geq 2.0$ or $\leq 0.5]$ and $t$-test $(\mathrm{P}<0.05)$. Gene Ontology $(\mathrm{GO})$ and Kyoto Encyclopedia of Genes and Genomes (KEGG) pathway enrichment analyses were performed using public databases.

\section{Statistical analysis}

All data were expressed as mean \pm SD. Statistical significance was assessed by the paired $t$-test or one-way analysis of 
variance (ANOVA) between two groups and multiple groups, respectively. Statistical significance was considered when $\mathrm{P}<0.05$. All statistical analysis was carried out using the statistical software GraphPad Prism 8 (GraphPad Software Inc., La Jolla, CA, USA).

\section{Results}

\section{RP11-465L10.10 is upregulated in TAA/D tissues and secretory VSMCs}

Previous studies have showed RP11-465L10.10 (also known as IncRNA SLC12A5-AS1) is highly related to the expression of MMP9, and participates in the progression of TAA/D $(12,13)$. Thus, the expression of RP11-465L10.10 and MMP9 was analyzed in aortic vascular wall tissues from TAD patients. The RT-qPCR, western blot, and immunohistochemistry results confirmed both RP11-465L10.10 and MMP9 were upregulated in TAD tissues (Figure 1A-1D), which indicated that $R P 11-465 L 10.10$ promotes TAD progression by regulating MMP9 expression.

The phenotype switching of VSMCs contributes to the development of TAA/D (19). To reveal the expression of RP11-465L10.10 in VSMCs phenotype switching in vitro, angiotensin II (Ang II) was subjected to induce human VSMCs transformed from contractile phenotype into synthetic ones (Figure $1 E, 1 F$ ). The results of RT-qPCR showed that RP11-465L10.10 and MMP9 were significantly increased in VSMCs treated with Ang II (Figure 1G,1H). In conclusion, all above results revealed that RP11-465L10.10 may participate in TAD by regulating VSMCs phenotype switching.

\section{Overexpression RP11-465L10.10 in VSMCs}

To investigate the functional roles and potential mechanisms of RP11-465L10.10 in VSMCs phenotype switching, an RP11-465L10.10 overexpressed lentivirus was produced. Briefly, the DNA fragment of RP11-465L10.10 was amplified and cloned into a $\mathrm{pCDH}$ lentivirus vector, and confirmed by gel electrophoresis after restriction enzyme digestion (Figure 2A). Then, the lentivirus particles were harvested and used to infect VSMCs. A flow cytometry sorting system was used to enrich the GFP positive cell ratio from $46.53 \%$ to $87.78 \%$ (Figure $2 B$ ). The expression of RP11-465L10.10 was measured by RT-qPCR, and the results showed that $R P 11-465 L 10.10$ was dramatically increased (more than 120 folds) in RP11-465L10.10(+)
VSMCs than control group. Meanwhile, RP11-465L10.10 knockdown was carried out in RP11-465L10.10(+) VSMCs by transfecting with $R P 11-465 L 10.10$ specific silencer (Figure 2C). The results of SM22 $\alpha$ and MMP9 expression results showed that SM22 $\alpha$ level decreased with RP11465 L10.10 overexpression, whereas the expression of MMP9 showed a consistent trend as RP11-465L10.10 (Figure 2D,2E). These data suggested that RP11-465L10.10 overexpression induced VSMCs phenotype switching and MMP9 expression.

\section{RP11-465L10.10 inducing VSMCs phenotype switching}

With the VSMCs phenotype transformed from contractile into secretory, their migration ability and proliferation ability were also enhanced (14). As shown in Figure $3 A$, the migration of RP11-465L10.10(+) VSMCs was promoted compared with the control group. Meanwhile, the enhanced migration capability was reversed after RP11-465L10.10 silenced. The EdU staining showed consistent alternation trend of proliferation in RP11-465L10.10 differently expressed VSMCs (Figure 3B). These findings suggest that RP11-465L10.10 induces the proliferation and migration of VSMCs.

Under normal physiological conditions, the proliferation and apoptosis of VSMCs were in a dynamic balance in vascular walls. The transformation of VSMCs to secretory type was accompanied by the enhancement of their antiapoptotic ability. Therefore, apoptosis of VSMCs were detected by flow cytometry, early apoptosis (Annexin V positive) and late apoptosis (both Annexin $\mathrm{V}$ and 7-AAD positive) were counted together. Flow cytometry results showed that overexpression of RP11-465L10.10 presented a significantly decreased apoptosis ratio compared with the control group and RP11-465L10.10 siRNA group (Figure 3C). Nuclear pyknosis is a typical characteristic of apoptosis. By nuclear staining, we also observed overexpression of RP11-465L10.10 can decrease apoptotic cells, which was reversed with RP11-465L10.10 knockdown in VSMCs (Figure 3D). Therefore, our studies indicated that RP11-465L10.10 overexpression remarkably promotes VSMCs migration, proliferation, and anti-apoptosis capabilities, and these could be reversed by RP11-465L10.10 knockdown.

\section{RP11-465L10.10 regulated VSMCs phenotype switching and MMP9 expression via the nuclear factor- $\mathrm{\kappa} B$ pathway}

To further ascertain the potential biological functions and 
A
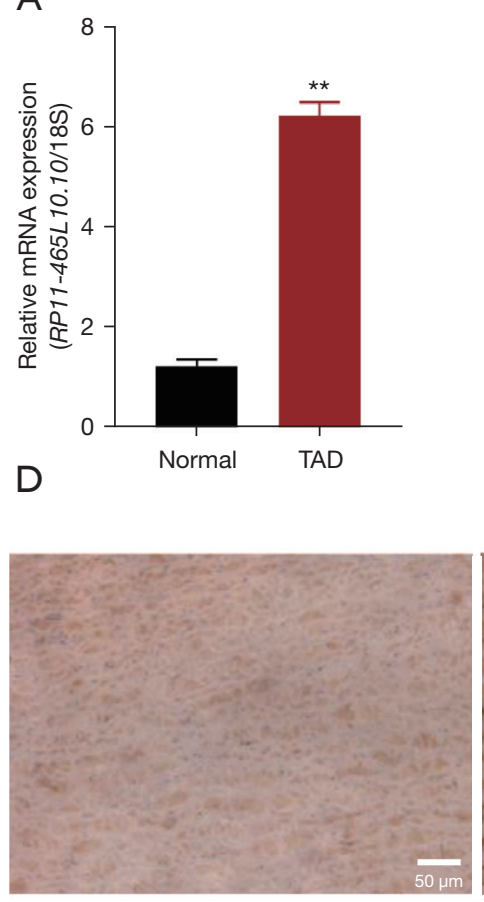

Normal

$\mathrm{F}$

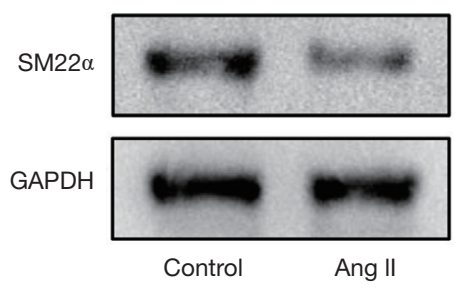

B
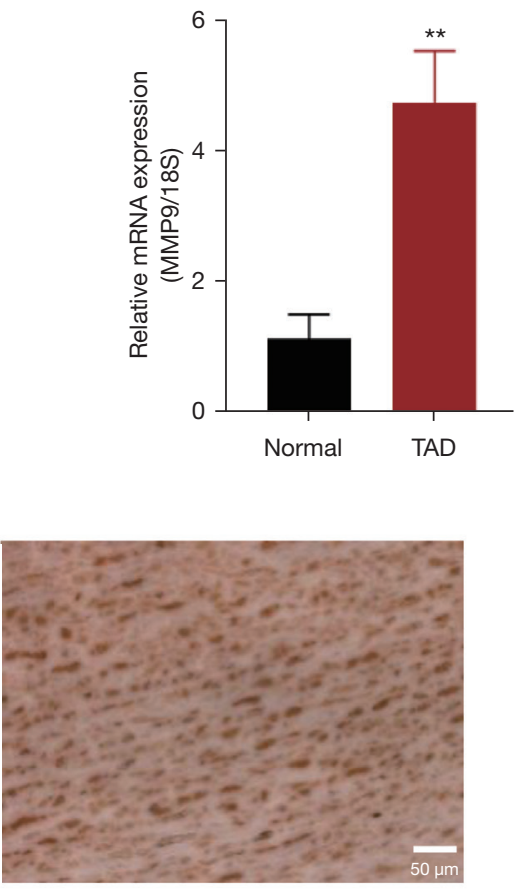

TAD

G

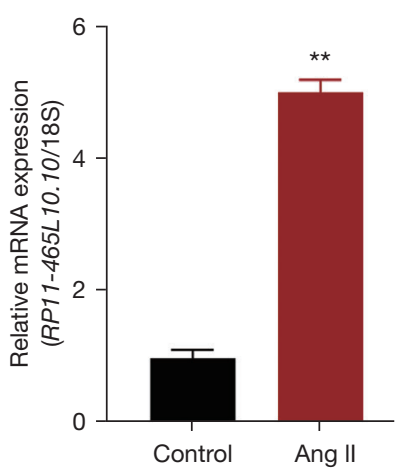

C

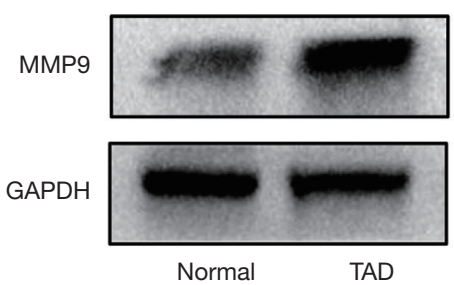

E

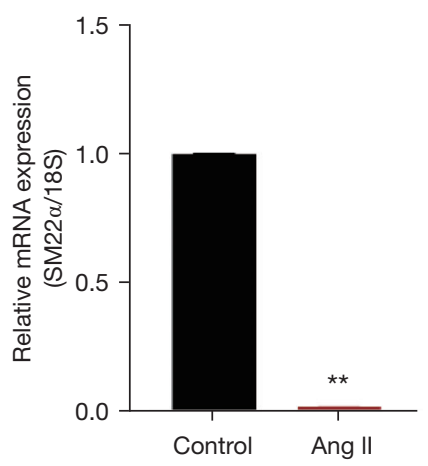

$\mathrm{H}$

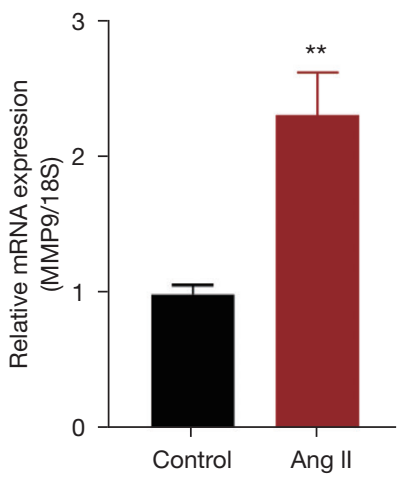

Figure 1 The expression of RP11-465L10.10 and MMP9 in TAD tissues and Ang II induced VSMCs in vitro. (A) The expression of RP11-465L10.10 was upregulated in TAD tissues; (B) RT-qPCR showed the expression level of MMP9 in TAD tissues was significantly upregulated; (C,D) Western blot and immunohistochemical staining showed the expression of MMP9 in TAD group was upregulated. Bar $=50 \mu \mathrm{m} ;(\mathrm{E}, \mathrm{F})$ the expression of SM22 $\alpha$ in Ang II induced VSMCs was significantly lower both in mRNA and protein levels; (G) RT-qPCR showed the expression of RP11-465L10.10 was significantly increased in Ang II induced VSMCs; (H) RT-qPCR showed the expression of $M M P 9$ was significantly increased in Ang II induced VSMCs. $\mathrm{n}=3$ for each group; ${ }^{* *}, \mathrm{P}<0.01$. TAD, thoracic aortic dissection; Ang II, angiotensin II; VSMC, vascular smooth muscle cell; RT-qPCR, reverse transcription quantitative polymerase chain reaction; mRNA, messenger RNA. 
A
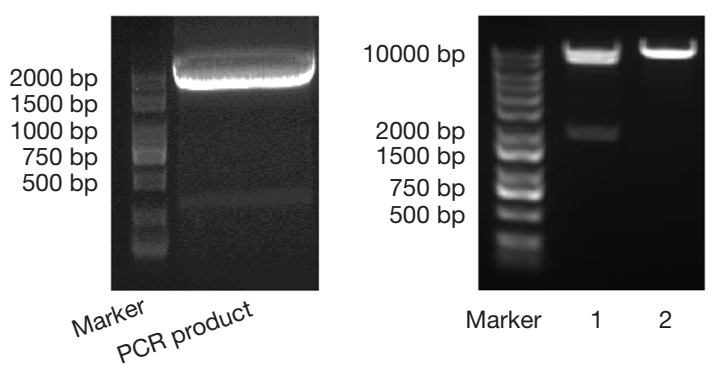

B

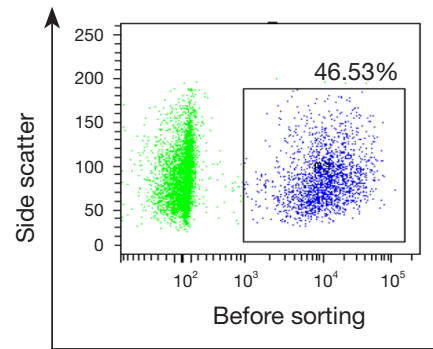

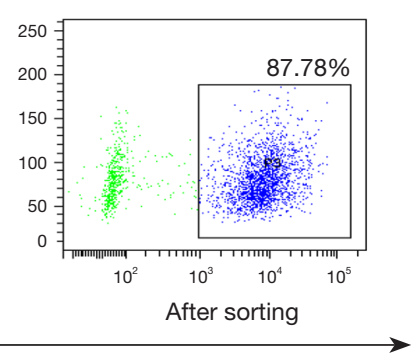

Green fluorescence
C

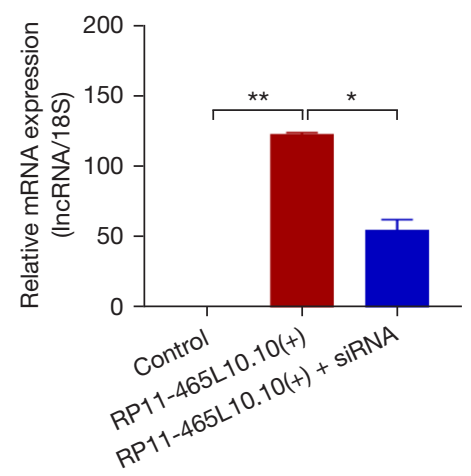

$\mathrm{E}$

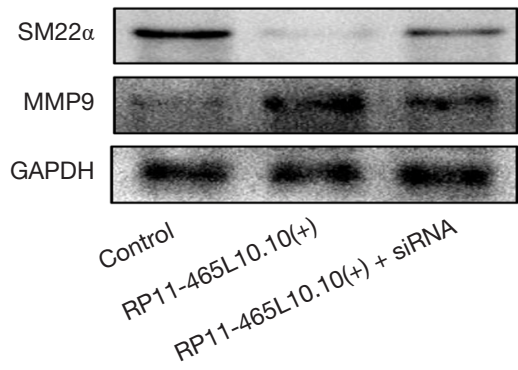

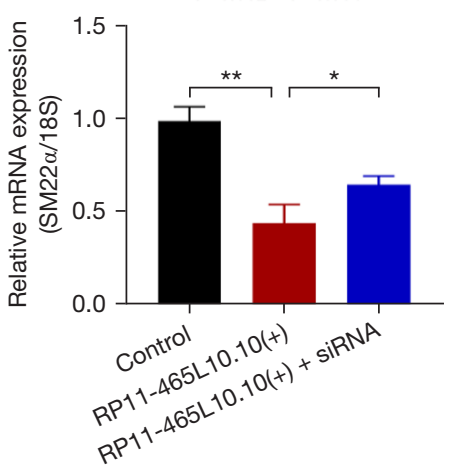

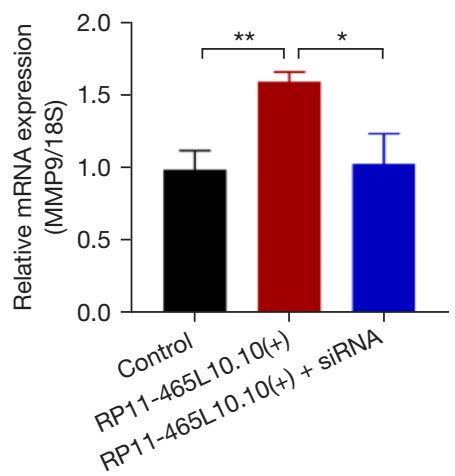

Figure 2 Overexpression RP11-465L10.10 in VSMCs. (A) Gel electrophoresis to identify RP11-465L10.10 DNA fragments. Lane1: RP11465L10.10 overexpression plasmid. Lane2: control plasmid; (B) flow cytometric analysis of green fluorescence positive VSMCs transfected with RP11-465L10.10 overexpressing lentivirus after sorting; (C) RT-qPCR results showed the expression of RP11-465L10.10 was significantly upregulated in RP11-465L10.10(+) VSMCs and reversed by transfecting with RP11-465L10.10 specific Silencer; (D) RT-qPCR showed the expression of SM22 $\alpha$ was down-regulated in RP11-465L10.10(+) VSMCs and reversed with RP11-465L10.10 inhibition; the expression of MMP9 showed a consistent trend to RP11-465L10.10 expression; (E) Western blot showed consistent trend with RT-qPCR results in protein level of $S M 22 \alpha$ and $M M P 9 . \mathrm{n}=3$ for each group; *, $\mathrm{P}<0.05$; **, $\mathrm{P}<0.01$. VSMC, vascular smooth muscle cell; RT-qPCR, reverse transcription quantitative polymerase chain reaction.

related signal pathways of $R P 11-465 L 10.10$ in VSMCs phenotypic switch, RNA-seq was performed to determine the differentially expressed genes with RP11-465L10.10 overexpression. The results showed there were 455 differentially expressed genes, including 199 up-regulated genes and 256 down-regulated genes (Figure $4 A$ ). In addition, hierarchical cluster analysis showed a difference in the messenger RNA (mRNA) expression profiles between RP11465L10.10(+) VSMCs and control group, such as NOTCH3 and SOX9, were upregulated in RP11-465L10.10(+) VSMCs, as previously reported (Figure 4B) $(20,21)$.

The results of GO functional enrichment analysis 
A

$\mathrm{Oh}$

$24 \mathrm{~h}$
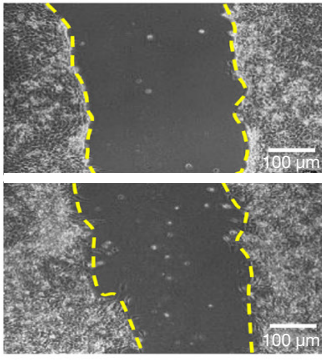

Control

B

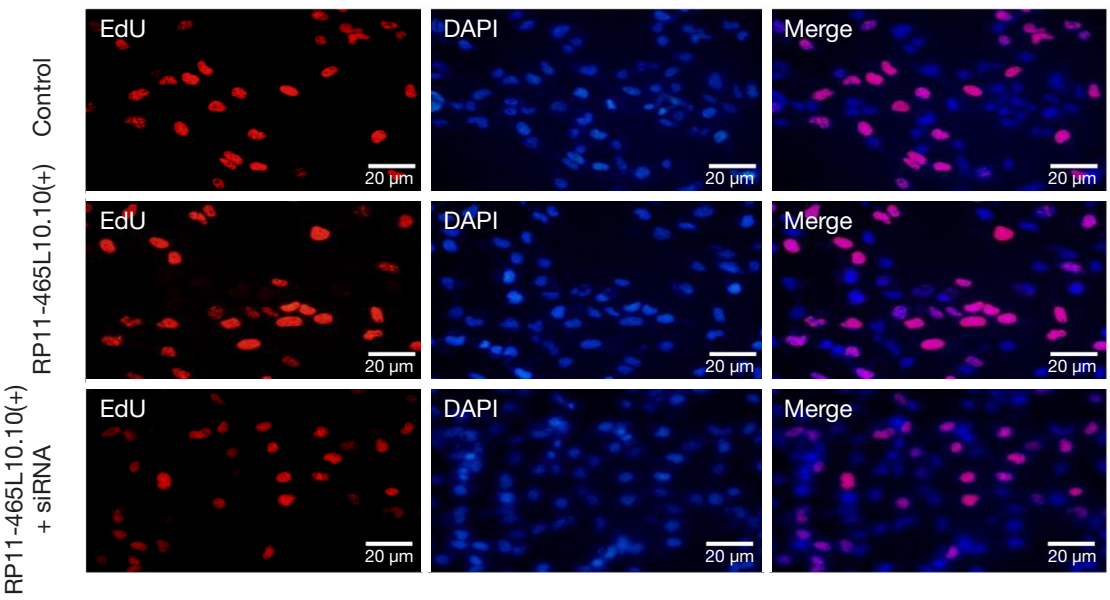

C

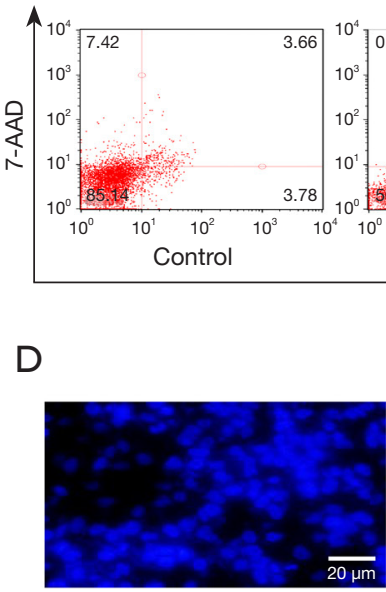

Control

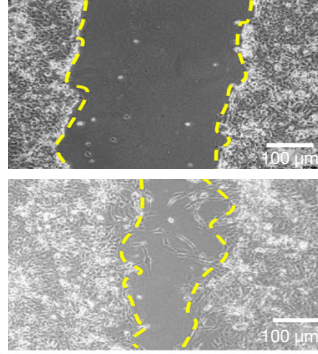

RP11-465L10.10(+)
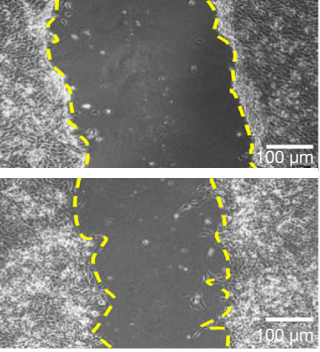

RP11-465L10.10(+) + siRNA
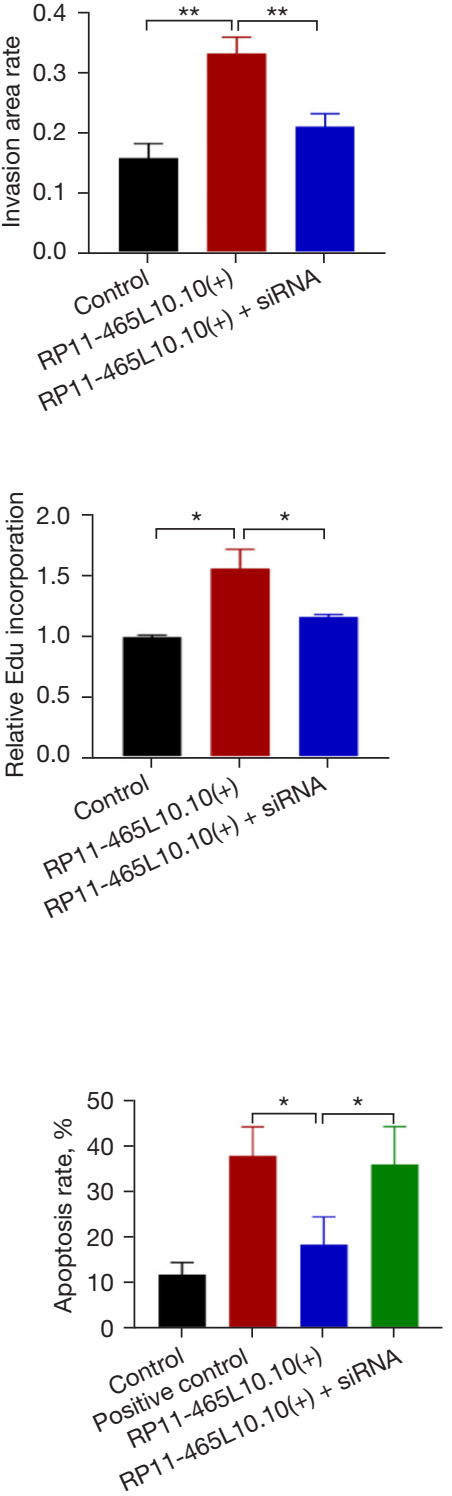

Annexin $\mathrm{V}-\mathrm{PE}$ RP

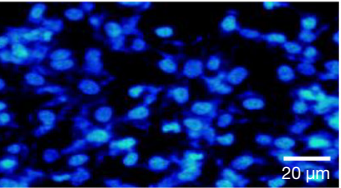

Positive control

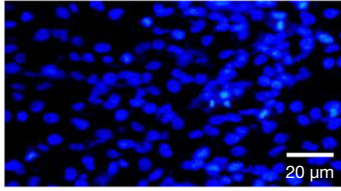

RP11-465L10.10(+)

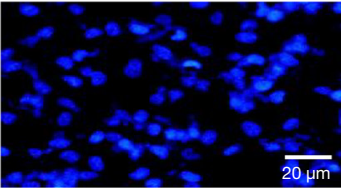

RP11-465L10.10(+) + siRNA

Figure 3 RP11-465L10.10 inducing VSMCs phenotype switching. (A) Wound healing migration assay showed the migration ability of VSMCs was enhanced with RP11-465L10.10 overexpression. Bar =100 $\mathrm{mm}$; (B) EdU incorporation assay showed the proliferation was increased in RP11-465L10.10(+) VSMCs and impaired with RP11-465L10.10 silencer. Bar =20 $\mu$; (C) flow cytometry analysis demonstrated the apoptotic ratio of VSMCs was decreased with RP11-465L10.10 overexpression; (D) nuclear staining also showed the RP11-465L10.10(+) VSMCs possess a lower apoptosis ratio with $\mathrm{H}_{2} \mathrm{O}_{2}$ treatment. Bar $=20 \mu \mathrm{m} . \mathrm{n}=3$ for each group; ${ }^{*}, \mathrm{P}<0.05 ;{ }^{* *}, \mathrm{P}<0.01$. VSMC, vascular smooth muscle cell. 
A
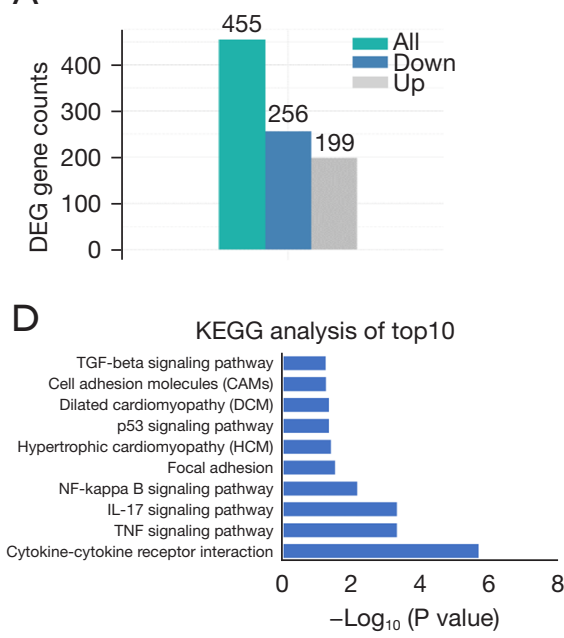

$E$

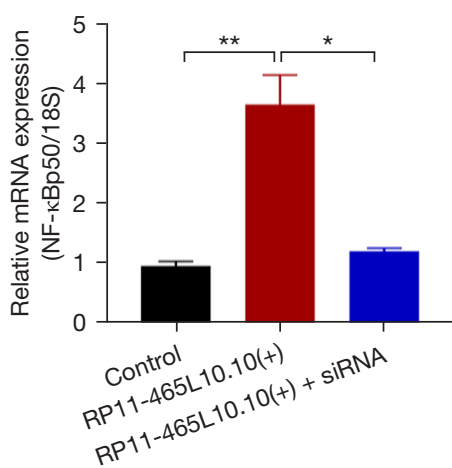

$\mathrm{F}$

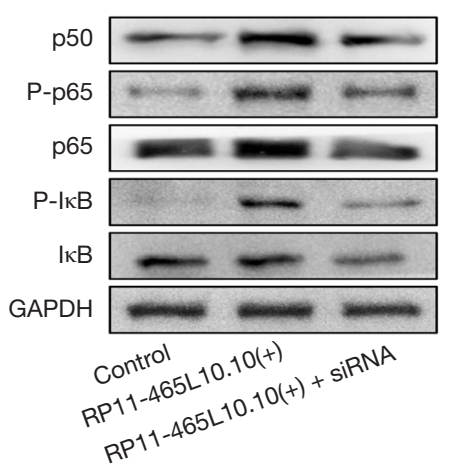

B
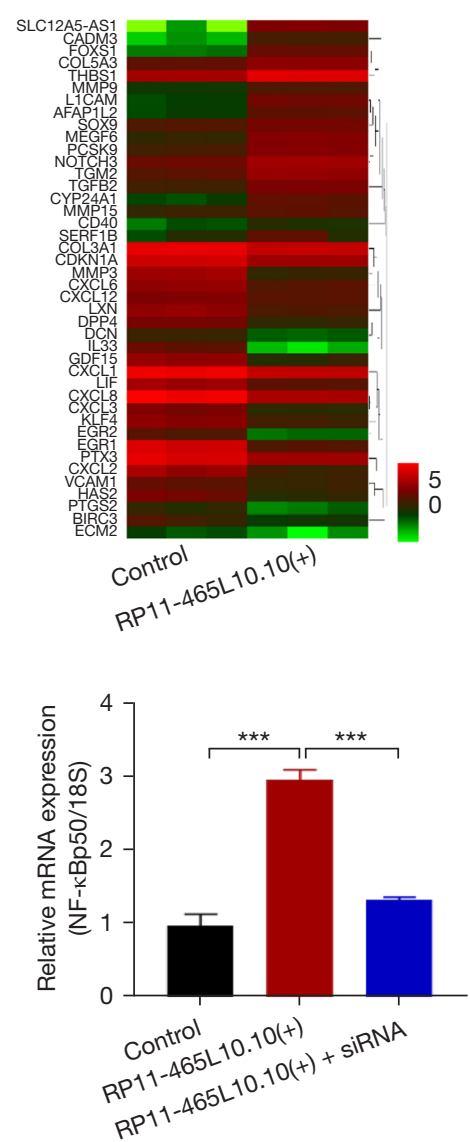

C
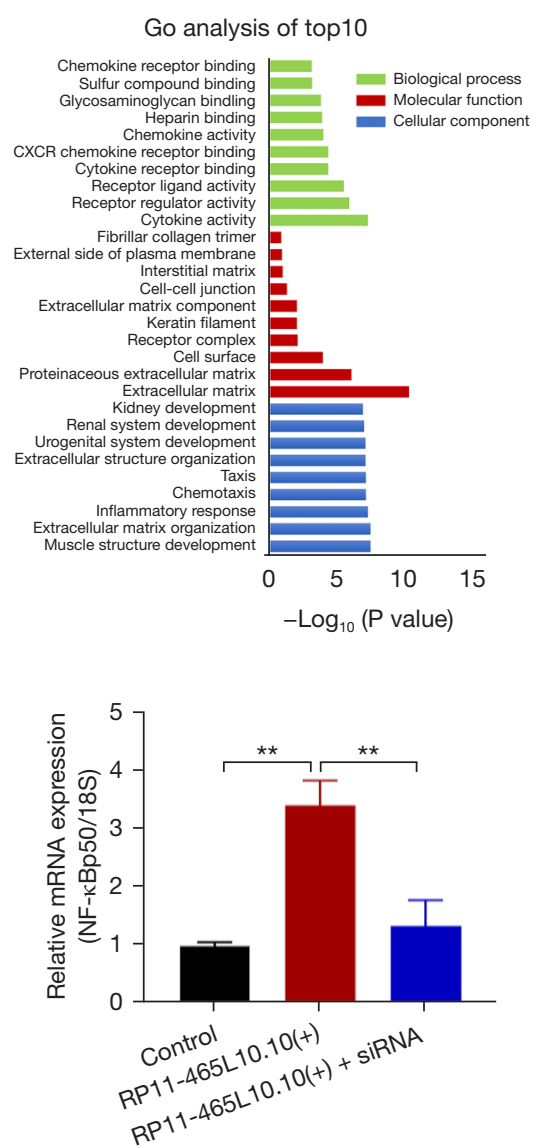

G

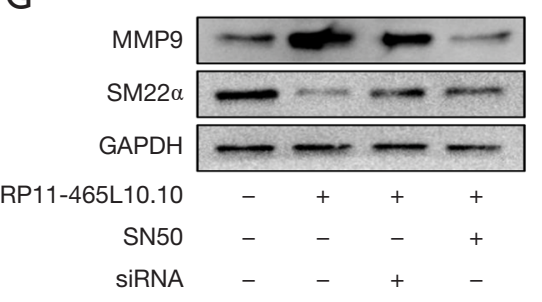

Figure 4 RP11-465L10.10 regulated VSMCs phenotype switching and MMP9 expression via the NF-kB pathway. (A) Histogram of the number of different genes between RP11-465L10.10(+) and control group. Blue column and gray column represent up-regulated and downregulated differential expressed genes respectively; (B) hierarchical cluster showed a difference in the mRNA expression profiles between overexpression RP11-465L10.10 VSMCs and control groups; (C) GO enrichment analysis histograms, which were drawn according to the 3 categories of biological processes, cellular components, and molecular functions; (D) KEGG pathway enrichment analysis of differently expressed genes; (E) RT-qPCR showed that the expression of p65, p50 and IкB were increased in RP11-465L10.10(+) group and inhibited with silencer transfected; (F) Western blot showed that the protein levels of p65, p50, and IкB in the RP11-465L10.10(+) group were upregulated; (G) the phenotypic switch and MMP9 expression were inhibited by inhibiting the NF-kB signal pathway in RP11-465L10.10(+) VSMCs. *, $\mathrm{P}<0.05$; ${ }^{* *}, \mathrm{P}<0.01$; ${ }^{* *}, \mathrm{P}<0.001$. VSMC, vascular smooth muscle cell; mRNA, messenger RNA; GO, Gene Ontology; KEGG, Kyoto Encyclopedia of Genes and Genomes; RT-qPCR, reverse transcription quantitative polymerase chain reaction. 
revealed several terms related to $R P 11-465 L 10.10$ in biological processes, cell composition, and molecular functions, among which ECM and cytokine activity are significantly enriched (Figure $4 C$ ). Additionally, for differentially expressed mRNAs, KEGG pathway analysis revealed that $R P 11-465 L 10.10$ regulates several different signaling pathways including the tumor necrosis factor (TNF) signaling pathway, interleukin (IL)-17 signaling pathway, and nuclear factor- $\kappa \mathrm{B}(\mathrm{NF}-\kappa \mathrm{B})$ signaling pathway (Figure 4D). Among these, the NF- $\kappa \mathrm{B}$ signal pathway participated the inflammatory phase of TAD as an important inflammatory stimulus for VSMCs phenotype switching $(22,23)$.

Previous studies have indicated that RP11-465L10.10 may facilitate NF- $\mathrm{\kappa B}$ p50 expression motif as a cis-acting element (13). Therefore, we used western blot to examine $\mathrm{NF}-\kappa \mathrm{B}$ p50 and some other key proteins involved in the $\mathrm{NF}-\kappa \mathrm{B}$ pathway. The results showed the expression of p50, P-p65, and P-IкB was significantly increased in RP11465 L10.10 overexpressed VSMCs (Figure 4E, 4F). We further used SN50 (a NF- $\kappa$ B signaling pathway inhibitor) to inhibit the activation of $\mathrm{NF}-\mathrm{\kappa B}$ signaling in RP11465L10.10(+) VSMCs (24). Moreover, we observed that the expression of $S M 22 \alpha$ increased and MMP9 decreased in SN50 groups (Figure 4G). Therefore, our above results demonstrated that NF- $\mathrm{\kappa B}$ signaling is necessary in RP11465L10.10-mediated VSMC phenotype transformation.

\section{Discussion}

The pathological section of TAA/D showed structural abnormalities of the aortic media (25), specifically manifested as rupture of elastin and disordered arrangement of collagen fibers in the media (26). As the main component of the aortic media, VSMCs are closely related to the function of the vascular wall (27). The role of contractile VSMCs is to maintain the structural stability of the aortic wall, functionally express contractile proteins and maintain the elasticity of the aorta. It has been demonstrated that VSMCs phenotypic modulation plays vital roles in the pathophysiologic processes of TAD (3). Switching of VSMCs from the contractile to secretory phenotype is an important biological behavior, which is marked with the enhanced proliferation, migration, and anti-apoptosis (28). The enhanced migration ability of secretory VSMCs promote the VSMCs migrated from the media to the intima. The apoptotic ability of secretory VSMCs declines, these VSMCs in the over-mature cell cycle are not enough to maintain structural and functional stability, and their anti-apoptotic ability increases, which leads to the selfclearance failure of senescent VSMCs (29). Further, reduced and weakened the elasticity and the contraction ability of secretory phenotype VSMCs, resulting weak aortic wall and leading to the occurrence of thoracic aortic dissection (30). Matrix metalloproteinases are important extracellular matrix components synthesized by VSMCs, and they regulate the structure and function of the extracellular matrix (31). Matrix metalloproteinases are a family of zinc-dependent endopeptidase enzymes, which are initially synthesized and secreted as precursor proteins. Secreted VSMCs are fully developed and mature cell states of VSMCs, and secrete a large amount of extracellular matrix such as MMP2 and MMP9, which results in the disorder of the media structure make it develop into a thoracic aortic dissection under various inducements such as hypertension changes (17).

In the present study, we found the expression of RP11465L10.10 and MMP9 were upregulated both in TAD aortic wall and Ang II-induced VSMCs phenotype switching model in vitro, which indicated that RP11465 L10.10 may function as a regulator in TAA/D vascular lesions. Abnormal migration and proliferation caused by VSMCs phenotypic switch are hallmarks for pathogenesis of TAA/D (32). In our current study, functional experiments performed in VSMCs revealed that the proliferation and migration of VSMCs was improved, and apoptosis was inhibited by increasing RP11-465L10.10 expression.

We performed RNA sequencing analysis to explore the target genes and regulated pathways of RP11465L10.10. The GO analysis showed that RP11-465L10.10 participates in multiple biological processes including muscle structure development and ECM organization, both of which are closely related to vascular contractile function. Excitingly, ECM-associated cellular component and molecular function motifs were enriched from GO analysis. These findings indicated that RP11-465L10.10 plays important roles in regulation of ECM. The MMP9 gene is one of primary enzymes responsible for the matrix degradation and disorganization. A previous study revealed that the expression of $M M P 9$ is significantly increased in aortic aneurysm (33). The overexpression of MMP9 and reduced level of $S M 22 \alpha$ impairs the elastin-contractile units of VSMCs (34-37). Our mechanical experiments also showed that RP11-465L10.10 induced MMP9 expression in VSMCs. In addition, the ECM such as elastic fibers, adhesion proteins, and other molecular changes are related to cell proliferation, adhesion, and migration. This may 
partially explain the enhanced migration and proliferation in RP11-465L10.10(+) VSMCs. All of these data demonstrated that RP11-465L10.10 promotes VSMCs phenotype changing and MMP9 expression, which are important promoting factors for TAA/D.

The KEGG pathway enrichment analysis of differentially expressed genes in the RP11-465L10.10(+) group was applied to determine the potential associated pathway. The KEGG analysis indicated that NF- $\kappa \mathrm{B}$ signaling was involved in RP11-465L10.10-induced VSMCs phenotypic switch. An abundance of evidence has demonstrated that $\mathrm{NF}-\kappa \mathrm{B}$ pathway activation leads to ECM degradation and VSMC phenotypic switch (38-42). Prediction of transcription factor binding sites indicated that RP11$465 \mathrm{~L} 10.10$ may be a cis-acting element to regulate $\mathrm{NF}$ $\kappa \mathrm{B}$ p50 expression. Theoretically, activation of the NF- $\kappa \mathrm{B}$ signal pathway would lead to an enhanced migration and proliferation. Western blot confirmed that the expressions of p50, P-p65, and P-I $\mathrm{B}$ were increased in VSMCs with RP11-465L10.10 upregulating (Figure 4F). Furthermore, when we interfered with $\mathrm{NF}-\kappa \mathrm{B}$ pathway activation in RP11-465L10.10(+) VSMCs with SN50, the effects of VSMCs phenotype switching induced by RP11-465L10.10 was impaired. Consistent with this, we first demonstrated RP11-465L10.10 promoting VSMCs phenotype switching and MMP9 expression via $\mathrm{NF}-\kappa \mathrm{B}$ signaling pathway.

\section{Conclusions}

It is crucial to identify the mechanisms that promote VSMCs phenotypic switch, with the aim of developing an effective pharmacologic treatment to prevent TAA/D progression. Taken together, our study found that RP11465 L10.10 presents at a high-level in TAD tissues, and aggravates the proliferation and migration of VSMCs. Our further investigations showed that RP11-465L10.10 promotes the VSMCs phenotype switch and MMP9 expression via the $\mathrm{NF}-\kappa \mathrm{B}$ signaling pathway. Most importantly, this research may lead to progress in lncRNA based therapies, and has revealed that RP11-465L10.10 might have potential therapeutic value for TAA/D treatment.

\section{Acknowledgments}

Authors would like to thank all of the patients who donated vascular tissues for use in this study.

Funding: This study was supported by the National Natural
Science Foundation of China (91839101, 82000434); Natural Science Foundation of Jiangsu Province (BK20200202).

\section{Footnote}

Reporting Checklist: The authors have completed the MDAR reporting checklist. Available at https://dx.doi. org/10.21037/atm-21-6402

Data Sharing Statement: Available at https://dx.doi. org/10.21037/atm-21-6402

Conflicts of Interest: All authors have completed the ICMJE uniform disclosure form (available at https://dx.doi. org/10.21037/atm-21-6402). The authors have no conflicts of interest to declare.

Ethical Statement: The authors are accountable for all aspects of the work in ensuring that questions related to the accuracy or integrity of any part of the work are appropriately investigated and resolved. The study was conducted in accordance with the Declaration of Helsinki (as revised in 2013). The study was approved by the Institutional Review Board of the First Affiliated Hospital of Soochow University (No. 2021-305). Written informed consents were provided by individual participants or their guardians.

Open Access Statement: This is an Open Access article distributed in accordance with the Creative Commons Attribution-NonCommercial-NoDerivs 4.0 International License (CC BY-NC-ND 4.0), which permits the noncommercial replication and distribution of the article with the strict proviso that no changes or edits are made and the original work is properly cited (including links to both the formal publication through the relevant DOI and the license). See: https://creativecommons.org/licenses/by-nc-nd/4.0/.

\section{References}

1. Tchana-Sato V, Sakalihasan N, Defraigne JO. Aortic dissection. Rev Med Liege 2018;73:290-5.

2. Gawinecka J, Schönrath F, von Eckardstein A. Acute aortic dissection: pathogenesis, risk factors and diagnosis. Swiss Med Wkly 2017;147:w14489.

3. Petsophonsakul P, Furmanik M, Forsythe R, et al. Role of Vascular Smooth Muscle Cell Phenotypic Switching and 
Calcification in Aortic Aneurysm Formation. Arterioscler Thromb Vasc Biol 2019;39:1351-68.

4. Yuan SM, Wu N. Aortic $\alpha$-smooth muscle actin expressions in aortic disorders and coronary artery disease: An immunohistochemical study. Anatol J Cardiol 2018;19:11-6.

5. Wang L, Zhang J, Fu W, et al. Association of smooth muscle cell phenotypes with extracellular matrix disorders in thoracic aortic dissection. J Vasc Surg 2012;56:1698709, 1709.e1.

6. Bridges MC, Daulagala AC, Kourtidis A. LNCcation: lncRNA localization and function. J Cell Biol 2021;220:e202009045.

7. Ransohoff JD, Wei Y, Khavari PA. The functions and unique features of long intergenic non-coding RNA. Nat Rev Mol Cell Biol 2018;19:143-57.

8. Jathar S, Kumar V, Srivastava J, et al. Technological Developments in lncRNA Biology. Adv Exp Med Biol 2017;1008:283-323.

9. Sun Y, Zhong L, He X, et al. LncRNA H19 promotes vascular inflammation and abdominal aortic aneurysm formation by functioning as a competing endogenous RNA. J Mol Cell Cardiol 2019;131:66-81.

10. Wu YX, Zhang SH, Cui J, et al. Long Noncoding RNA XR007793 Regulates Proliferation and Migration of Vascular Smooth Muscle Cell via Suppressing miR-23b. Med Sci Monit 2018;24:5895-903.

11. Zhang B, Dong Y, Zhao Z. LncRNA MEG8 regulates vascular smooth muscle cell proliferation, migration and apoptosis by targeting PPAR $\alpha$. Biochem Biophys Res Commun 2019;510:171-6.

12. Long $W$, Rui C, Song $X$, et al. Distinct expression profiles of $\operatorname{lncRNAs}$ between early-onset preeclampsia and preterm controls. Clin Chim Acta 2016;463:193-9.

13. Li Y, Yang N. Microarray expression profile analysis of long non-coding RNAs in thoracic aortic aneurysm. Kaohsiung J Med Sci 2018;34:34-42.

14. Gomez D, Owens GK. Smooth muscle cell phenotypic switching in atherosclerosis. Cardiovasc Res 2012;95:156-64.

15. Cui N, Hu M, Khalil RA. Biochemical and Biological Attributes of Matrix Metalloproteinases. Prog Mol Biol Transl Sci 2017;147:1-73.

16. Olszyński K, Zimowska M. Structure and function of matrix metalloproteinases. Postepy Biochem 2009;55:76-84.

17. An Z, Liu Y, Song ZG, et al. Mechanisms of aortic dissection smooth muscle cell phenotype switch. J Thorac Cardiovasc Surg 2017;154:1511-1521.e6.

18. Grada A, Otero-Vinas M, Prieto-Castrillo F, et al.
Research Techniques Made Simple: Analysis of Collective Cell Migration Using the Wound Healing Assay. J Invest Dermatol 2017;137:e11-6.

19. Shen M, Hu M, Fedak PWM, et al. Cell-Specific Functions of ADAM17 Regulate the Progression of Thoracic Aortic Aneurysm. Circ Res 2018;123:372-88.

20. Qiao L, Xie L, Shi K, et al. Notch signaling change in pulmonary vascular remodeling in rats with pulmonary hypertension and its implication for therapeutic intervention. PLoS One 2012;7:e51514.

21. Augstein A, Mierke J, Poitz DM, et al. Sox9 is increased in arterial plaque and stenosis, associated with synthetic phenotype of vascular smooth muscle cells and causes alterations in extracellular matrix and calcification. Biochim Biophys Acta Mol Basis Dis 2018;1864:2526-37.

22. Zhong L, He X, Si X, et al. SM22 $\alpha$ (Smooth Muscle 22 $\alpha$ )

Prevents Aortic Aneurysm Formation by Inhibiting Smooth Muscle Cell Phenotypic Switching Through Suppressing

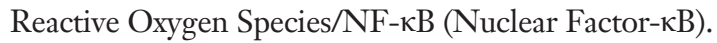
Arterioscler Thromb Vasc Biol 2019;39:e10-25.

23. Lawrence $T$. The nuclear factor NF-kappaB pathway in inflammation. Cold Spring Harb Perspect Biol 2009;1:a001651.

24. Orlandi A, Francesconi A, Marcellini M, et al. PropionylL-carnitine reduces proliferation and potentiates Baxrelated apoptosis of aortic intimal smooth muscle cells by modulating nuclear factor-kappaB activity. J Biol Chem 2007;282:4932-42.

25. Graham DJ, Alexander JJ, Miguel R. Aortic endothelial and smooth muscle cell co-culture: an in vitro model of the arterial wall. J Invest Surg 1991;4:487-94.

26. Wagenseil JE, Mecham RP. Vascular extracellular matrix and arterial mechanics. Physiol Rev 2009;89:957-89.

27. Baumann F, Makaloski V, Diehm N. Aortic aneurysms and aortic dissection: epidemiology, pathophysiology and diagnostics. Internist (Berl) 2013;54:535-42.

28. Zhang J, Wang L, Fu W, et al. Smooth muscle cell phenotypic diversity between dissected and unaffected thoracic aortic media. J Cardiovasc Surg (Torino) 2013;54:511-21.

29. Zhang L, Cheng H, Yue Y, et al. H19 knockdown suppresses proliferation and induces apoptosis by regulating miR-148b/WNT/ $\beta$-catenin in ox-LDL -stimulated vascular smooth muscle cells. J Biomed Sci 2018;25:11.

30. Afewerki T, Ahmed S, Warren D. Emerging regulators of vascular smooth muscle cell migration. J Muscle Res Cell Motil 2019;40:185-96. 
31. Johnson JL. Metalloproteinases in atherosclerosis. Eur J Pharmacol 2017;816:93-106.

32. Wang Y, Dong CQ, Peng GY, et al. MicroRNA-134-5p Regulates Media Degeneration through Inhibiting VSMC Phenotypic Switch and Migration in Thoracic Aortic Dissection. Mol Ther Nucleic Acids 2019;16:284-94.

33. Maguire EM, Pearce SWA, Xiao R, et al. Matrix Metalloproteinase in Abdominal Aortic Aneurysm and Aortic Dissection. Pharmaceuticals (Basel) 2019;12:118.

34. Wang X, Li D, Chen H, et al. Expression of Long Noncoding RNA LIPCAR Promotes Cell Proliferation, Cell Migration, and Change in Phenotype of Vascular Smooth Muscle Cells. Med Sci Monit 2019;25:7645-51.

35. Qu C, Liu X, Guo Y, et al. MiR-128-3p inhibits vascular smooth muscle cell proliferation and migration by repressing FOXO4/MMP9 signaling pathway. Mol Med 2020;26:116.

36. Montes de Oca A, Madueño JA, Martinez-Moreno JM, et al. High-phosphate-induced calcification is related to SM22 $\alpha$ promoter methylation in vascular smooth muscle cells. J Bone Miner Res 2010;25:1996-2005.

37. Shu YN, Zhang F, Bi W, et al. SM $22 \alpha$ inhibits vascular inflammation via stabilization of $\mathrm{I} \kappa \mathrm{B} \alpha$ in vascular smooth muscle cells. J Mol Cell Cardiol 2015;84:191-9.

38. Zhang L, Liu L, Bai M, et al. Hypoxia-induced HE4 in tubular epithelial cells promotes extracellular matrix accumulation and renal fibrosis via NF- $\kappa$ B. FASEB J 2020;34:2554-67.

39. Rigoglou S, Papavassiliou AG. The NF- $\mathrm{BB}$ signalling pathway in osteoarthritis. Int J Biochem Cell Biol 2013;45:2580-4.

40. Thenappan T, Chan SY, Weir EK. Role of extracellular matrix in the pathogenesis of pulmonary arterial hypertension. Am J Physiol Heart Circ Physiol 2018;315:H1322-31.

41. Kong P, Yu Y, Wang L, et al. circ-Sirt1 controls NF$\kappa \mathrm{B}$ activation via sequence-specific interaction and enhancement of SIRT1 expression by binding to miR132/212 in vascular smooth muscle cells. Nucleic Acids Res 2019;47:3580-93.

42. Lu QB, Wan MY, Wang PY, et al. Chicoric acid prevents PDGF-BB-induced VSMC dedifferentiation, proliferation and migration by suppressing ROS/NFкB/mTOR/ P70S6K signaling cascade. Redox Biol 2018;14:656-68.

(English Language Editor: J. Jones)
Cite this article as: Lin $\mathrm{Y}$, Huang H, Yu Y, Zhu F, Xiao W, Yang Z, Shao L, Shen Z. Long non-coding RNA RP11-465L10.10 promotes vascular smooth muscle cells phenotype switching and MMP9 expression via the NF- $\mathrm{B}$ pathway. Ann Transl Med 2021;9(24):1776. doi: 10.21037/atm21-6402 
Supplementary

Table S1 The sequence of primers used in this study

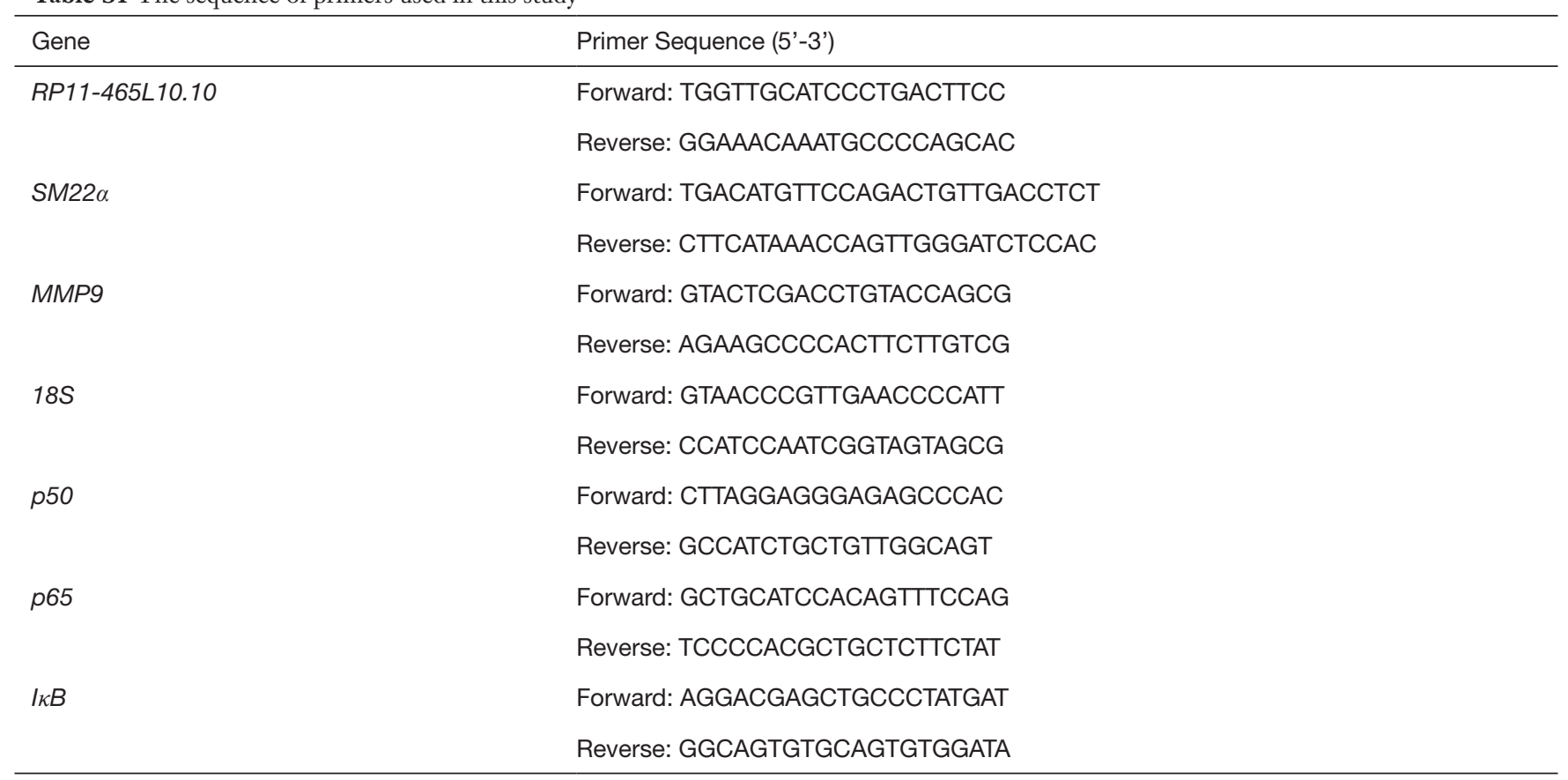

